\title{
On a forward-backward stochastic system associated to the Burgers equation
}

\author{
Ana Bela Cruzeiro ${ }^{1,2}$ and Evelina Shamarova ${ }^{3}$ \\ ${ }^{1}$ Dep. de Matemática, IST-UTL. \\ ${ }^{2}$ Grupo de Física Matemática da Universidade de Lisboa. \\ E-mail: abcruz@math.ist.utl.pt \\ ${ }^{3}$ Centro de Matemática da Universidade do Porto. \\ E-mail: evelinas@fc.up.pt
}

\begin{abstract}
We describe a probabilistic construction of $H^{\alpha}$-regular solutions for the spatially periodic forced Burgers equation by using a characterization of this solution through a forward-backward stochastic system.
\end{abstract}

\section{Introduction}

Burgers equation, given by

$$
\partial_{s} y(s, \theta)+(y, \nabla) y(s, \theta)-\nu \Delta y(s, \theta)+F(s, \theta)=0
$$

is sometimes presented as a simplified model for turbulence and describes the motion of a compressible fluid with viscosity $\nu$ under the influence of a force $F$. In this paper we establish a connection between a time-changed spatially periodic Burgers equation and a forward-backward stochastic system on the group of diffeomorphisms of a torus, similar to the characterization we have studied in [C-S] for the incompressible (Navier-Stokes equation) case.

It is well known that forward-backward systems are closely related to partial differential equations. For references in the subject one can use for example those in $[\mathrm{D}]$ or in the more recent work [C-S-T-V]. One difference in 
our approach is that the stochastic processes are defined in the group of diffeomorphisms of the underlying configuration space of the fluid (in our case, the torus) and not in the configuration space itself. This group is endowed with a Sobolev topology. The importance of working with infinite dimensional geometry, in the line of thought introduced by V. Arnold ([A]) for the Euler equation is, partly, that it allows to construct solutions which are "automatically" Sobolev regular in the space variable. Also, which is more important, the geometric objects defined in the (infinite dimensional) path spaces may allow to prove several properties of the dynamics, such as stability for the corresponding flows.

Generally speaking, our approach can be regarded as a stochastic deformation of the Lagrangian picture keeping the "mean velocity" (the Eulerian picture) of the motion unchanged. This means that the mean velocity, given by the drift, is still the relevant deterministic solution of the (velocity) equations of motion. This approach finds its roots in the works [N-Y-Z], [Y]. It is the point of view described in $[\mathrm{G}]$, but is completely different from the approaches that consist in perturbing the Eulerian (velocity) dynamics by a random noise.

In [C-S] we have developed in this spirit a construction for the NavierStokes equations. We derived a solution of the stochastic system from a solution of Navier-Stokes equation and, "reciprocally", defined Navier-Stokes solutions from the stochastic forward-backward flows. The incompressibility condition there makes the geometry much more delicate to study then in the present Burgers case and, in this respect, the Burgers equation is a simplification of the framework of [C-S]. On the other hand here we prove an existence result of the stochastic forward-backward equation (without assuming the existence of the p.d.e. solution), a result which is not proved for the Navier-Stokes case. This is therefore the main result of this paper and the one that really distinguishes it from [C-S]. The methods we use to prove this result are close to those of Delarue in [D]. Therefore we obtain here a completely probabilistic construction for the Burgers solutions. We treat the torus case since it is one of the simplest compact manifolds; the results, with the necessary adjustments, should extend to other manifolds.

We refer to [C-S] and references therein for the general framework of the stochastic approach to partial differential equations such as Burgers and Navier-Stokes that we are dealing with. 


\section{The main result}

Let us consider the spatially periodic backward Burgers equation in $\mathbb{R}^{n}$ :

$$
\left\{\begin{array}{l}
\partial_{s} y(s, \theta)+(y, \nabla) y(s, \theta)+\nu \Delta y(s, \theta)+F(s, \theta)=0 \\
y(T, \theta)=h(\theta) .
\end{array}\right.
$$

It is obtained from the classical Burgers equation by means of the substitution $u(t, \theta) \leftrightarrow-u(T-t, \theta)$, where $T>0$ is fixed arbitrary. We assume that $h$ belongs to the Sobolev space of order $\alpha, H^{\alpha}\left(\mathbb{T}^{n}, \mathbb{R}^{n}\right)$ and that $F$ belongs to $H^{\alpha}\left(\mathbb{T}^{n}, \mathbb{R}^{n}\right)$, where $\alpha$ is bigger than $\frac{n}{2}+2$. The symbol $\mathbb{T}^{n}$ denotes the $n$-dimensional torus, namely $\mathbb{T}^{n}=\underbrace{S^{1} \times \ldots \times S^{1}}_{n}$, and $S^{1}$ is a unit circle. We extend the functions $F$ and $h$ to $\mathbb{R}^{n}$ periodically, and use the same symbols for the extended functions. Consider the following system of forward-backward SDEs in $H^{\alpha}\left(\mathbb{T}^{n}, \mathbb{R}^{n}\right)$ :

$$
\left\{\begin{array}{l}
d Z_{s}^{t, e}=Y_{s}^{t, e} d s+\sqrt{2 \nu} d W_{s} \\
d Y_{s}^{t, e}=-F(s, \cdot) \circ Z_{s}^{t, e} d s+\sqrt{2 \nu} X_{s}^{t, e} d W_{s} \\
Z_{t}^{t, e}=e ; Y_{T}^{t, e}=h \circ Z_{T}^{t, e}
\end{array}\right.
$$

where $e: \mathbb{T}^{n} \rightarrow \mathbb{T}^{n}$ is the identical map, $W_{s}$ is an $n$-dimensional Brownian motion and $\nu>0$. Let $\mathcal{F}_{s}=\sigma\left(W_{r}, r \in[0, s]\right)$. We would like to find an $\mathcal{F}_{s}$-adapted triple of stochastic processes $\left(Z_{s}^{t, e}, Y_{s}^{t, e}, X_{s}^{t, e}\right)$ with values in $H^{\alpha}\left(\mathbb{T}^{n}, \mathbb{R}^{n}\right) \times H^{\alpha}\left(\mathbb{T}^{n}, \mathbb{R}^{n}\right) \times H^{\alpha}\left(\mathbb{T}^{n}, \mathbb{R}^{n \times n}\right)$ which solves $(2)$. Note that the process $X_{s}^{t, e}$ takes values in the space of linear operators $\mathcal{L}\left(\mathbb{R}^{n}, H^{\alpha}\left(\mathbb{T}^{n}, \mathbb{R}^{n}\right)\right)$ $\left(\approx H^{\alpha}\left(\mathbb{T}^{n}, \mathbb{R}^{n \times n}\right)\right)$, i.e.

$$
X_{s}^{t, e}=\sum_{i=1}^{n} X_{s}^{i} \otimes e_{i}
$$

where the processes $X_{s}^{i}$ take values in $H^{\alpha}\left(\mathbb{T}^{n}, \mathbb{R}^{n}\right)$, and $\left\{e_{i}\right\}_{i=1}^{n}$ is an orthonormal basis of $\mathbb{R}^{n}$. Define

$$
K=\sup _{\mathbb{T}^{n}}|\nabla h|+\sup _{[0, T] \times \mathbb{T}^{n}}|\nabla F|
$$

where $T$ is the arbitrary fixed number that we used to obtain the backward Burgers equation. Our main result is the following. 
Theorem 1. Let $h \in H^{\alpha}\left(\mathbb{T}^{n}, \mathbb{R}^{n}\right), F(s, \cdot) \in H^{\alpha}\left(\mathbb{T}^{n}, \mathbb{R}^{n}\right), s \in[0, T]$, be such that $\nabla^{l} h$ and $\nabla^{l} F(s, \cdot)$ are bounded for all $l \leqslant \alpha$ and for all $s \in[0, T]$. Then, there exists $a T_{0}>0$ that depends only on $\alpha$ and $K$ defined by (4) and such that for every $T<T_{0}$ and for every $t \in[0, T]$, there exists a unique $\mathcal{F}_{s^{-}}$ adapted solution $\left(Z_{s}^{t, e}, Y_{s}^{t, e}, X_{s}^{t, e}\right)$ to (2) on $[t, T]$ with values in $H^{\alpha}\left(\mathbb{T}^{n}, \mathbb{R}^{n}\right) \times$ $H^{\alpha}\left(\mathbb{T}^{n}, \mathbb{R}^{n}\right) \times H^{\alpha}\left(\mathbb{T}^{n}, \mathbb{R}^{n \times n}\right)$. Moreover, the function $y:[0, T] \times \mathbb{T}^{n} \rightarrow \mathbb{R}^{n}$, $(t, \theta) \mapsto Y_{t}^{t, e}(\theta)$ is deterministic, and solves the problem (1).

First we prove the existence and uniqueness of an $\mathcal{F}_{s}$-adapted $H^{\alpha}\left(\mathbb{T}^{n}, \mathbb{R}^{n}\right) \times H^{\alpha}\left(\mathbb{T}^{n}, \mathbb{R}^{n}\right) \times H^{\alpha}\left(\mathbb{T}^{n}, \mathbb{R}^{n \times n}\right)$-valued solution $\left(Z_{s}^{t, \xi}, Y_{s}^{t, \xi}, X_{s}^{t, \xi}\right)$ to the following problem:

$$
\begin{aligned}
Z_{s}^{t, \xi} & =\xi+\int_{t}^{s} Y_{r}^{t, \xi} d r+\sqrt{2 \nu}\left(W_{s}-W_{t}\right), \\
Y_{s}^{t, \xi} & =h \circ Z_{T}^{t, \xi}+\int_{s}^{T} F(r, \cdot) \circ Z_{r}^{t, \xi} d r-\sqrt{2 \nu} \int_{s}^{T} X_{r}^{t, \xi} d W_{r},
\end{aligned}
$$

where $\xi$ is an $\mathcal{F}_{t^{-}}$-measurable $H^{\alpha}\left(\mathbb{T}^{n}, \mathbb{R}^{n}\right)$-valued random variable. Without loss of generality we will assume that the derivatives $\nabla^{l} h$ and $\nabla^{l} F(t, \cdot), t \in$ $[0, T]$, are everywhere defined. In the following, we will identify $H^{0}\left(\mathbb{T}^{n}, \mathbb{R}^{n}\right)$ and $L_{2}\left(\mathbb{T}^{n}, \mathbb{R}^{n}\right)$. The proof of Theorem 1 will be devided in several lemmas.

LEMMA 1. Let $\mathbb{E}\|\xi\|_{L_{p}\left(\mathbb{T}^{n}, \mathbb{R}^{n}\right)}^{p}$ and $\mathbb{E}\left\|\nabla^{i} \xi\right\|_{L_{p}\left(\mathbb{T}^{n}, \mathbb{R}^{n^{i}}\right)}^{p}$ be bounded for all integers $i \leqslant l$ and $p \geqslant 2$. Further suppose that the FBSDEs (5), (6) have a solution in $H^{l}\left(\mathbb{T}^{n}, \mathbb{R}^{n}\right)$. Then for any integer $p \geqslant 2$ there exists a $T_{0}>0$ that depends only on $p$ and $K$ defined by (4) and such that for all positive $T<T_{0}$, for all $i \leqslant l, \mathbb{E}\left\|Z_{s}^{t, \xi}\right\|_{L_{p}\left(\mathbb{T}^{n}, \mathbb{R}^{n}\right)}^{p}$ and $\mathbb{E}\left\|\nabla^{i} Z_{s}^{t, \xi}\right\|_{L_{p}\left(\mathbb{T}^{n}, \mathbb{R}^{n^{i}}\right)}^{p}$ are bounded on $[t, T]$.

Proof. Everywhere below, for convenience, we use the same symbols $\gamma$ and $\gamma_{i}, i=1,2,3 \ldots$, for (different) constants in different formulas. All $\gamma$ and $\gamma_{i}$ below are positive and do not depend on $s \in[t, T]$ and $\theta \in \mathbb{T}^{n}$. Note that, for any Hilbert norm,

$$
\begin{aligned}
& \left(\|g\|^{p}\right)^{\prime} h=p\|g\|^{p-2}(g, h), \\
& \left(\|g\|^{p}\right)^{\prime \prime} h_{1} h_{2}=p(p-2)\|g\|^{p-4}\left(g, h_{1}\right)\left(g, h_{2}\right)+p\|g\|^{p-2}\left(h_{1}, h_{2}\right) .
\end{aligned}
$$

Fix a $\theta \in \mathbb{T}^{n}$, and let $z_{s}=Z_{s}^{t, \xi}(\theta), y_{s}=Y_{s}^{t, \xi}(\theta), x_{s}=X_{s}^{t, \xi}(\theta)$. BSDE (6) and 
Itô's formula imply:

$$
\begin{gathered}
\mathbb{E}\left|y_{s}\right|^{p}-\sqrt{2 \nu} p(p-2) \int_{s}^{T} \mathbb{E}\left[\left|y_{r}\right|^{p-4} \sum_{i=1}^{n}\left|\left(x_{r}^{i}, y_{r}\right)\right|^{2}\right] d r \\
-\sqrt{2 \nu} p \int_{s}^{T} \mathbb{E}\left[\left|y_{r}\right|^{p-2}\left|x_{r}\right|^{2}\right] d r=\mathbb{E}\left|h\left(z_{T}\right)\right|^{p}+2 p \int_{s}^{T} \mathbb{E}\left[\left|y_{r}\right|^{p-2}\left(F\left(r, z_{r}\right), y_{r}\right)\right] d r
\end{gathered}
$$

where $x_{r}^{i}=X_{r}^{i}(\theta)$ and the processes $X_{r}^{i}$ were introduced in representation (3). Taking into account that $h$ and $F$ are bounded on $\mathbb{T}^{n}$ and resp. $[0, T] \times \mathbb{T}^{n}$, and applying Young's inequality we obtain the existence of constants $\gamma_{1}$ and $\gamma_{2}$ such that

$$
\mathbb{E}\left|y_{s}\right|^{p} \leqslant \gamma_{1}+\gamma_{2} \int_{s}^{T} \mathbb{E}\left|y_{r}\right|^{p} d r .
$$

Applying Gronwall's lemma and then integrating over $\mathbb{T}^{n}$ we obtain that there exists a constant $\gamma$ such that

$$
\mathbb{E}\left\|Y_{s}^{t, \xi}\right\|_{L_{p}\left(\mathbb{T}^{n}, \mathbb{R}^{n}\right)}^{p} \leqslant \gamma
$$

From SDE (5), we deduce the existence of constants $\gamma_{1}$ and $\gamma_{2}$ such that

$$
\mathbb{E}\left|z_{s}\right|^{p} \leqslant \gamma_{1} \mathbb{E}|\xi|^{p}+\gamma_{2} \int_{t}^{s} \mathbb{E}\left|y_{r}\right|^{p} d r
$$

Integrating over $\mathbb{T}^{n}$ and modyfing $\gamma$ we obtain:

$$
\mathbb{E}\left\|Z_{s}^{t, \xi}\right\|_{L_{p}\left(\mathbb{T}^{n}, \mathbb{R}^{n}\right)}^{p} \leqslant \gamma
$$

Let us prove now that $\mathbb{E}\left\|\nabla Z_{s}^{t, \xi}\right\|_{L_{p}\left(\mathbb{T}^{n}, \mathbb{R}^{n}\right)}^{p}$ is bounded, where $\nabla$ stands, as usual, for the space derivative. The triple $\left(\nabla Z_{s}^{t, \xi}, \nabla Y_{s}^{t, \xi}, \nabla X_{s}^{t, \xi}\right)$ solves the FBSDEs:

$$
\begin{aligned}
& \nabla Z_{s}^{t, \xi}=\nabla \xi+\int_{t}^{s} \nabla Y_{r}^{t, \xi} d r \\
& \nabla Y_{s}^{t, \xi}=\nabla h\left(\left(Z_{T}^{t, \xi}(\cdot)\right) \nabla Z_{T}^{t, \xi}+\int_{s}^{T} \nabla F\left(r, Z_{r}^{t, \xi}(\cdot)\right) \nabla Z_{r}^{t, \xi} d r-\sqrt{2 \nu} \int_{s}^{T} \nabla X_{r}^{t, \xi} d W_{r} .\right.
\end{aligned}
$$


For simplicity of the notation, let us introduce the processes $z_{s}=Z_{s}^{t, \xi}(\theta)$, $y_{s}=Y_{s}^{t, \xi}(\theta)$ and $x_{s}=X_{s}^{t, \xi}(\theta)$. Itô's formula together with the BSDE (8) imply:

$$
\begin{gathered}
\left|\nabla y_{s}\right|^{p}+\sqrt{2 \nu} p(p-2) \int_{s}^{T}\left|\nabla y_{r}\right|^{p-4} \sum_{i=1}^{n}\left|\left(\nabla x_{r}^{i}, \nabla y_{r}\right)\right|^{2} d r \\
+\sqrt{2 \nu} p \int_{s}^{T}\left|\nabla y_{s}\right|^{p-2}\left|\nabla x_{r}\right|^{2} d r=\left|\nabla h\left(z_{T}\right) \nabla z_{T}\right|^{p} \\
+2 p \int_{s}^{T}\left|\nabla y_{r}\right|^{p-2}\left(\nabla F\left(r, z_{r}\right) \nabla z_{r}, \nabla y_{r}\right) d r+2 p \int_{s}^{T}\left|\nabla y_{r}\right|^{p-2}\left(\nabla y_{r}, \nabla x_{r} d W_{r}\right) .
\end{gathered}
$$

This implies that there exist constants $\gamma_{1}, \gamma_{2}$, and $\gamma_{3}$ such that for all $s \in$ $[t, T]$

$$
\mathbb{E}\left|\nabla y_{s}\right|^{p} \leqslant \gamma_{1} \mathbb{E}|\nabla \xi|^{p}+\gamma_{2} \mathbb{E} \int_{t}^{T}\left|\nabla y_{r}\right|^{p-2}\left|\nabla z_{r}\right|^{2} d r+\gamma_{3} \int_{t}^{T} \mathbb{E}\left|\nabla y_{r}\right|^{p} d r
$$

From Young's inequality,

$$
\left|\nabla y_{r}\right|^{p-2}\left|\nabla z_{r}\right|^{2} \leqslant \frac{p-2}{p}\left|\nabla y_{r}\right|^{p}+\frac{2}{p}\left|\nabla z_{r}\right|^{p} .
$$

Therefore, we can find constants $\gamma_{1}$ and $\gamma_{2}$ such that

$$
\mathbb{E}\left|\nabla y_{s}\right|^{p} \leqslant \gamma_{1} \mathbb{E}|\nabla \xi|^{p}+\gamma_{2} \int_{t}^{T} \mathbb{E}\left|\nabla y_{r}\right|^{p} d r
$$

Choosing $T_{0}$ smaller than $\frac{1}{\gamma_{2}}$ we deduce that there exists a constant $\gamma$ such that

$$
\mathbb{E}\left|\nabla y_{s}\right|^{p} \leqslant \gamma \mathbb{E}|\nabla \xi|^{p}
$$

Integrating over $\mathbb{T}^{n}$ gives:

$$
\mathbb{E}\left\|\nabla Y_{s}^{t, \xi}\right\|_{L_{p}\left(\mathbb{T}^{n}, \mathbb{R}^{\left.n^{2}\right)}\right.}^{p} \leqslant \gamma \mathbb{E}\|\nabla \xi\|_{L_{p}\left(\mathbb{T}^{n}, \mathbb{R}^{n^{2}}\right)}^{p} .
$$

Next, the SDE (7) implies that there exist positive constants $\gamma_{3}$ and $\gamma_{4}$ such that

$$
\mathbb{E}\left\|\nabla Z_{s}^{t, \xi}\right\|_{L_{p}\left(\mathbb{T}^{n}, \mathbb{R}^{n^{2}}\right)}^{p} \leqslant \gamma_{3} \mathbb{E}\|\nabla \xi\|_{L_{p}\left(\mathbb{T}^{n}, \mathbb{R}^{n^{2}}\right)}^{p}+\gamma_{4} \int_{t}^{s} \mathbb{E}\left\|\nabla Y_{r}^{t, \xi}\right\|_{L_{p}\left(\mathbb{T}^{n}, \mathbb{R}^{n^{2}}\right)}^{p} d r .
$$


Combining this and (10) and modifying $\gamma$ and $\gamma_{1}$ we obtain:

$$
\mathbb{E}\left\|\nabla Z_{s}^{t, \xi}\right\|_{L_{p}\left(\mathbb{T}^{n}, \mathbb{R}^{\left.n^{2}\right)}\right.}^{p} \leqslant \gamma_{1} \mathbb{E}\|\nabla \xi\|_{L_{p}\left(\mathbb{T}^{n}, \mathbb{R}^{n^{2}}\right)}^{p} \leqslant \gamma
$$

Let us assume $\mathbb{E}\left\|\nabla^{i} Z_{s}^{t, \xi}\right\|_{L_{p}\left(\mathbb{T}^{n}, \mathbb{R}^{\left.n^{2}\right)}\right.}^{p} \leqslant \gamma$ for all integers $i \leqslant l-1$, and prove that $\mathbb{E}\left\|\nabla^{l} Z_{s}^{t, \xi}\right\|_{L_{p}\left(\mathbb{T}^{n}, \mathbb{R}^{n^{2}}\right)}^{p} \leqslant \gamma$ The triple $\left(\nabla^{l} Z_{s}^{t, \xi}, \nabla^{l} Y_{s}^{t, \xi}, \nabla^{l} X_{s}^{t, \xi}\right)$ solves the FBSDEs (11), (12) below which are obtain from (5), (6) by differentiating both parts $l$ times:

$$
\begin{aligned}
& \nabla^{l} Z_{s}^{t, \xi}=\nabla^{l} \xi+\int_{t}^{s} \nabla^{l} Y_{r}^{t, \xi} d r \\
& \nabla^{l} Y_{s}^{t, \xi}=\nabla h\left(Z_{T}^{t, \xi}(\cdot)\right) \nabla^{l} Z_{T}^{t, \xi}+\int_{s}^{T} \nabla F\left(r, Z_{r}^{t, \xi}(\cdot)\right) \nabla^{l} Z_{r}^{t, \xi} d r \\
& +\sum_{j=2}^{l} \nabla^{j} h\left(Z_{T}^{t, \xi}(\cdot)\right)\left[\sum_{i_{1}+\cdots+i_{j}=l-j+1} \nabla^{i_{1}} Z_{T}^{t, \xi} \ldots \nabla^{i_{j}} Z_{T}^{t, \xi}\right] \\
& +\int_{s}^{T} \sum_{j=2}^{l} \nabla^{j} F\left(r, Z_{r}^{t, \xi}(\cdot)\right)\left[\sum_{i_{1}+\cdots+i_{j}=l-j+1} \nabla^{i_{1}} Z_{r}^{t, \xi} \ldots \nabla^{i_{j}} Z_{r}^{t, \xi}\right] d r \\
& -\sqrt{2 \nu} \int_{s}^{T} \nabla^{l} X_{r}^{t, \xi} d W_{r} .
\end{aligned}
$$

The argument below is similar to the one we have used for the first order derivatives. Itô's formula and the BSDE (12) imply:

$$
\begin{gathered}
\left|\nabla^{l} y_{s}\right|^{p}+\sqrt{2 \nu} p \int_{s}^{T}\left|\nabla^{l} y_{s}\right|^{p-2}\left|\nabla^{l} x_{r}\right|^{2} d r \\
\quad+\sqrt{2 \nu} p(p-2) \int_{s}^{T}\left|\nabla^{l} y_{r}\right|^{p-4} \sum_{i=1}^{n}\left|\left(\nabla^{l} x_{r}^{i}, \nabla^{l} y_{r}\right)\right|^{2} d r \\
=\left|\nabla h\left(z_{T}\right) \nabla^{l} z_{T}+\sum_{j=2}^{l} \nabla^{j} h\left(z_{T}\right)\left[\sum_{i_{1}+\cdots+i_{j}=l-j+1} \nabla^{i_{1}} z_{T} \ldots \nabla^{i_{j}} z_{T}\right]\right|^{p} \\
+2 p \int_{s}^{T}\left|\nabla^{l} y_{r}\right|^{p-2}\left(\nabla F\left(r, z_{r}\right) \nabla^{l} z_{r}, \nabla^{l} y_{r}\right) d r+2 p \int_{s}^{T}\left|\nabla^{l} y_{r}\right|^{p-2}\left(\nabla^{l} y_{r}, \nabla^{l} x_{r} d W_{r}\right) \\
+\int_{s}^{T}\left|\nabla^{l} y_{r}\right|^{p-2}\left(\sum_{j=2}^{l} \nabla^{j} F\left(r, z_{r}\right)\left[\sum_{i_{1}+\cdots+i_{j}=l-j+1} \nabla^{i_{1}} z_{r} \ldots \nabla^{i_{j}} z_{r}\right], \nabla^{l} y_{r}\right) d r .
\end{gathered}
$$


Note that by (11), there exist constants $\gamma_{1}$ and $\gamma_{2}$ so that

$$
\left|\nabla^{l} z_{s}\right|^{p} \leqslant \gamma_{1}\left|\nabla^{l} \xi\right|^{p}+\gamma_{2} \int_{t}^{s}\left|\nabla^{l} y_{r}\right|^{p} d r
$$

This and (13) imply for all $s \in[t, T]$ there exist constants $\gamma_{3}, \gamma_{4}, \gamma_{5}, \gamma_{6}$, and $\gamma_{7}$ such that

$$
\begin{gathered}
\mathbb{E}\left|\nabla^{l} y_{s}\right|^{p} \leqslant \gamma_{3} \mathbb{E}\left|\nabla^{l} \xi\right|^{p}+\gamma_{4} \mathbb{E} \int_{t}^{T}\left|\nabla^{l} y_{r}\right|^{p-2}\left|\nabla^{l} z_{r}\right|^{2} d r+\gamma_{5} \int_{t}^{T} \mathbb{E}\left|\nabla^{l} y_{r}\right|^{p} d r \\
+\gamma_{6} \sum_{i_{1}+\cdots+i_{j} \leqslant l-1} \mathbb{E}\left|\nabla^{i_{1}} z_{T} \ldots \nabla^{i_{j}} z_{T}\right|^{p} \\
+\gamma_{7} \mathbb{E} \int_{t}^{T}\left|\nabla^{l} y_{r}\right|^{p-2}\left|\sum_{j=2}^{l} \nabla^{j} F\left(r, z_{r}\right)\left[\sum_{i_{1}+\ldots+i_{j}=l-j+1} \nabla^{i_{1}} z_{r} \ldots \nabla^{i_{j}} z_{r}\right]\right|^{2} d r
\end{gathered}
$$

Note that we can apply inequality (9) where $\nabla y_{r}$ is replaced by $\nabla^{l} y_{r}$ and $\nabla z_{r}$ is replaced by $\nabla^{l} z_{r}, r \in[t, T]$. Also, Young's inequality implies that there exists a constant $\gamma_{8}$ such that

$$
\begin{aligned}
\left|\nabla^{l} y_{r}\right|^{p-2} \mid \sum_{j=2}^{l} \nabla^{j} F\left(r, z_{r}\right) & {\left.\left[\sum_{i_{1}+\cdots+i_{j}=l-j+1} \nabla^{i_{1}} z_{r} \ldots \nabla^{i_{j}} z_{r}\right]\right|^{2} } \\
\leqslant \frac{p-2}{p}\left|\nabla^{l} y_{r}\right|^{p}+\frac{2}{p} \mid & \left.\sum_{j=2}^{l} \nabla^{j} F\left(r, z_{r}\right)\left[\sum_{i_{1}+\cdots+i_{j}=l-j+1} \nabla^{i_{1}} z_{r} \ldots \nabla^{i_{j}} z_{r}\right]\right|^{p} \\
& \leqslant \frac{p-2}{p}\left|\nabla^{l} y_{r}\right|^{p}+\gamma_{8} \sum_{i_{1}+\cdots+i_{j} \leqslant l-1}\left|\nabla^{i_{1}} z_{r} \ldots \nabla^{i_{j}} z_{r}\right|^{p} .
\end{aligned}
$$

Finally we obtain that there exist constants $\gamma_{1}, \gamma_{2}, \gamma_{3}$, and $\gamma_{4}$ such that

$$
\begin{aligned}
& \mathbb{E}\left|\nabla^{l} y_{s}\right|^{p} \leqslant \gamma_{1} \mathbb{E}\left|\nabla^{l} \xi\right|^{p}+\gamma_{2} \sum_{i_{1}+\cdots+i_{j} \leqslant l-1} \mathbb{E}\left|\nabla^{i_{1}} z_{T} \ldots \nabla^{i_{j}} z_{T}\right|^{p} \\
&+\gamma_{3} \int_{t}^{T} \sum_{i_{1}+\cdots+i_{j} \leqslant l-1} \mathbb{E}\left|\nabla^{i_{1}} z_{r} \ldots \nabla^{i_{j}} z_{r}\right|^{p} d r+\gamma_{4} \int_{t}^{T} \mathbb{E}\left|\nabla^{l} y_{r}\right|^{p} d r .
\end{aligned}
$$


Choosing $T_{0}$ smaller than $\frac{1}{\gamma_{4}}$ and modifying $\gamma_{1}, \gamma_{2}$ and $\gamma_{3}$ we obtain that

$$
\begin{aligned}
\mathbb{E}\left|\nabla^{l} y_{s}\right|^{p} \leqslant \gamma_{1} \mathbb{E}\left|\nabla^{l} \xi\right|^{p}+\gamma_{2} & \sum_{i_{1}+\cdots+i_{j} \leqslant l-1} \mathbb{E}\left|\nabla^{i_{1}} z_{T} \ldots \nabla^{i_{j}} z_{T}\right|^{p} \\
& +\gamma_{3} \int_{t}^{T} \sum_{i_{1}+\cdots+i_{j} \leqslant l-1} \mathbb{E}\left|\nabla^{i_{1}} z_{r} \ldots \nabla^{i_{j}} z_{r}\right|^{p} d r
\end{aligned}
$$

and moreover, modifying $\gamma_{1}, \gamma_{2}$ and $\gamma_{3}$ we obtain that

$$
\begin{aligned}
\mathbb{E}\left|\nabla^{l} z_{s}\right|^{p} \leqslant \gamma_{1} \mathbb{E}\left|\nabla^{l} \xi\right|^{p}+\gamma_{2} & \sum_{i_{1}+\cdots+i_{j} \leqslant l-1} \mathbb{E}\left|\nabla^{i_{1}} z_{T} \ldots \nabla^{i_{j}} z_{T}\right|^{p} \\
& +\gamma_{3} \int_{t}^{T} \sum_{i_{1}+\cdots+i_{j} \leqslant l-1} \mathbb{E}\left|\nabla^{i_{1}} z_{r} \ldots \nabla^{i_{j}} z_{r}\right|^{p} d r .
\end{aligned}
$$

Integrating over $\mathbb{T}^{n}$ and taking into account that $\mathbb{E}\left\|\nabla^{l} \xi\right\|_{L_{p}\left(\mathbb{T}^{n}, \mathbb{R}^{n^{l}}\right)}^{p}$ is bounded by assumption, $\mathbb{E}\left\|\nabla^{i_{1}} Z_{r}^{t, \xi} \ldots \nabla^{i_{j}} Z_{r}^{t, \xi}\right\|_{\left.L_{p}\left(\mathbb{T}^{n}, \mathbb{R}^{n}\right)^{l}\right)}^{p}, r \in[t, T]$, are bounded by Hölder's inequality and the induction hypothesis, we obtain that $\mathbb{E}\left\|\nabla^{l} Y_{s}^{t, \xi}\right\|_{L_{p}\left(\mathbb{T}^{n}, \mathbb{R}^{n}\right)}^{p}$ and $\mathbb{E}\left\|\nabla^{l} Z_{s}^{t, \xi}\right\|_{L_{p}\left(\mathbb{T}^{n}, \mathbb{R}^{\left.n^{l}\right)}\right.}^{p}$ are bounded.

LEMma 2. There exists a $T_{0}>0$ such that for every positive $T<T_{0}$ and for every $t \in[0, T]$, FBSDEs (5), (6) has a unique $\mathcal{F}_{s}$-adapted solution on $[t, T]$ with values in $H^{\alpha}\left(\mathbb{T}^{n}, \mathbb{R}^{n}\right) \times H^{\alpha}\left(\mathbb{T}^{n}, \mathbb{R}^{n}\right) \times H^{\alpha}\left(\mathbb{T}^{n}, \mathbb{R}^{n \times n}\right)$.

Proof. First we prove the existence of solution in $L_{2}\left(\mathbb{T}^{n}, \mathbb{R}^{n}\right)$. Let us consider the map

$$
\Gamma: L_{2}\left(\Omega, L_{2}\left(\mathbb{T}^{n}, \mathbb{R}^{n}\right)\right) \rightarrow L_{2}\left(\Omega, L_{2}\left(\mathbb{T}^{n}, \mathbb{R}^{n}\right)\right), Y_{s}^{t, \xi} \rightarrow \bar{Y}_{s}^{t, \xi}
$$

which is defined by the FBSDEs below:

$$
\begin{aligned}
& \bar{Z}_{s}^{t, \xi}=\xi+\int_{t}^{s} Y_{r}^{t, \xi} d r+\sqrt{2 \nu}\left(W_{s}-W_{t}\right), \\
& \bar{Y}_{s}^{t, \xi}=h\left(\bar{Z}_{T}^{t, \xi}(\cdot)\right)+\int_{s}^{T} F\left(r, \bar{Z}_{r}^{t, \xi}(\cdot)\right) d r-\sqrt{2 \nu} \int_{s}^{T} \bar{X}_{r}^{t, \xi} d W_{r} .
\end{aligned}
$$

First we find $\bar{Z}_{s}^{t, \xi}$ from the SDE (16), and substitute it into BSDE (17). Then we find the unique $\mathcal{F}_{s}$-adapted solution $\left(\bar{Y}_{s}^{t, \xi}, \bar{X}_{s}^{t, \xi}\right)$ of BSDE (17). Namely,

$$
\bar{Y}_{s}^{t, \xi}=\mathbb{E}\left[h\left(\bar{Z}_{T}^{t, \xi}(\cdot)\right)+\int_{s}^{T} F\left(r, \bar{Z}_{r}^{t, \xi}(\cdot)\right) d r \mid \mathcal{F}_{s}\right],
$$


and $\bar{X}_{s}^{t, \xi}$ exists by the martingale representation theorem (see [C-S]). Note that since $h$ and $F$ are bounded on $\mathbb{T}^{n}$ and resp. on $[0, T] \times \mathbb{T}^{n}, \bar{Y}_{s}^{t, \xi}$ takes values in $L_{2}\left(\mathbb{T}^{n}, \mathbb{R}^{n}\right)$. The process $\bar{X}_{s}^{t, \xi}$ is actually not needed for the definition of the map $\Gamma$. Let us prove that the map $\Gamma$ is a contraction. Let $V_{s}^{t, \xi}, Y_{s}^{t, \xi}$ $\in L_{2}\left(\Omega, L_{2}\left(\mathbb{T}^{n}, \mathbb{R}^{n}\right)\right)$, and $\bar{V}_{s}^{t, \xi}=\Gamma\left(V_{s}^{t, \xi}\right), \bar{Y}_{s}^{t, \xi}=\Gamma\left(Y_{s}^{t, \xi}\right)$. Further let $\bar{U}_{s}^{t, \xi}$ be obtained from (16), i.e. $\bar{U}_{s}^{t, \xi}=\xi+\int_{t}^{s} V_{r}^{t, \xi} d r+\sqrt{2 \nu}\left(W_{s}-W_{t}\right)$, and $\bar{W}_{s}^{t, \xi}$ be obtained from the BSDE (17), i.e. $\bar{V}_{s}^{t, \xi}=h\left(\bar{U}_{T}^{t, \xi}(\cdot)\right)+\int_{s}^{T} F\left(r, \bar{U}_{r}^{t, \xi}(\cdot)\right) d r-$ $\sqrt{2 \nu} \int_{s}^{T} \bar{W}_{r}^{t, \xi} d W_{r}$. The SDE (16) implies that for all $s \in[t, T]$

$$
\left\|\bar{Z}_{s}^{t, \xi}-\bar{U}_{s}^{t, \xi}\right\|_{L_{2}\left(\mathbb{T}^{n}, \mathbb{R}^{n}\right)}^{2} \leqslant(s-t) \int_{t}^{s}\left\|Y_{r}^{t, \xi}-V_{r}^{t, \xi}\right\|_{L_{2}\left(\mathbb{T}^{n}, \mathbb{R}^{n}\right)}^{2} d r .
$$

The SDE (17) and Itô's formula imply that

$$
\begin{aligned}
& \mathbb{E}\left\|\bar{Y}_{s}^{t, \xi}-\bar{V}_{s}^{t, \xi}\right\|_{L_{2}\left(\mathbb{T}^{n}, \mathbb{R}^{n}\right)}^{2}+2 \nu \int_{s}^{T} \mathbb{E}\left\|\bar{X}_{r}^{t, \xi}-\bar{W}_{r}^{t, \xi}\right\|_{L_{2}\left(\mathbb{T}^{n}, \mathbb{R}^{n}\right)}^{2} d r \\
& =\mathbb{E}\left\|h\left(\bar{Z}_{T}^{t, \xi}(\cdot)\right)-h\left(\bar{U}_{T}^{t, \xi}(\cdot)\right)\right\|_{L_{2}\left(\mathbb{T}^{n}, \mathbb{R}^{n}\right)}^{2} \\
& +2 \int_{s}^{T} \mathbb{E}\left(F\left(r, \bar{Z}_{r}^{t, \xi}(\cdot)\right)-F\left(r, \bar{U}_{T}^{t, \xi}(\cdot)\right), \bar{Y}_{r}^{t, \xi}-\bar{V}_{r}^{t, \xi}\right)_{L_{2}\left(\mathbb{T}^{n}, \mathbb{R}^{n}\right)} d r .
\end{aligned}
$$

Hence,

$$
\begin{aligned}
\mathbb{E}\left\|\bar{Y}_{s}^{t, \xi}-\bar{V}_{s}^{t, \xi}\right\|_{L_{2}\left(\mathbb{T}^{n}, \mathbb{R}^{n}\right)}^{2} \leqslant \mathbb{E}\left\|h\left(\bar{Z}_{T}^{t, \xi}(\cdot)\right)-h\left(\bar{U}_{T}^{t, \xi}(\cdot)\right)\right\|_{L_{2}\left(\mathbb{T}^{n}, \mathbb{R}^{n}\right)}^{2} \\
+\int_{t}^{T} \mathbb{E}\left\|F\left(r, \bar{Z}_{r}^{t, \xi}(\cdot)\right)-F\left(r, \bar{U}_{r}^{t, \xi}(\cdot)\right)\right\|_{L_{2}\left(\mathbb{T}^{n}, \mathbb{R}^{n}\right)}^{2} d r \\
+\int_{s}^{T} \mathbb{E}\left\|\bar{Y}_{r}^{t, \xi}-\bar{V}_{r}^{t, \xi}\right\|_{L_{2}\left(\mathbb{T}^{n}, \mathbb{R}^{n}\right)}^{2} d r .
\end{aligned}
$$

Gronwall's lemma and inequality (18) imply that

$$
\mathbb{E}\left\|\bar{Y}_{s}^{t, \xi}-\bar{V}_{s}^{t, \xi}\right\|_{L_{2}\left(\mathbb{T}^{n}, \mathbb{R}^{n}\right)}^{2} \leqslant \tilde{\gamma}(T) \int_{t}^{T} \mathbb{E}\left\|Y_{r}^{t, \xi}-V_{r}^{t, \xi}\right\|_{L_{2}\left(\mathbb{T}^{n}, \mathbb{R}^{n}\right)}^{2} d r
$$

where $\tilde{\gamma}(T)=e^{T} K$ and $K$ is defined by (4). Let us pick $T_{0}$ so that $\gamma\left(T_{0}\right)=$ $T_{0} \tilde{\gamma}\left(T_{0}\right)<1$. Then, if $T<T_{0}$,

$$
\sup _{s \in[t, T]} \mathbb{E}\left\|\bar{Y}_{s}^{t, \xi}-\bar{V}_{s}^{t, \xi}\right\|_{L_{2}\left(\mathbb{T}^{n}, \mathbb{R}^{n}\right)} \leqslant \gamma(T) \sup _{s \in[t, T]} \mathbb{E}\left\|Y_{s}^{t, \xi}-V_{s}^{t, \xi}\right\|_{L_{2}\left(\mathbb{T}^{n}, \mathbb{R}^{n}\right)} .
$$


This proves that for $T<T_{0}$ there is a unique fixed point $Y_{s}^{t, \xi}$ of the map $\Gamma$. The processes $Z_{s}^{t, \xi}$ and $X_{s}^{t, \xi}$ can be determined from (6) as described above. Let us consider now the FBSDEs which is obtained from (5), (6) by differentiation with respect to $\theta \in \mathbb{T}^{n}$ :

$$
\left\{\begin{array}{l}
\nabla Z_{s}^{t, \xi}=\nabla \xi+\int_{t}^{s} \nabla Y_{r}^{t, \xi} d r \\
\nabla Y_{s}^{t, \xi}=\nabla h\left(Z_{T}^{t, \xi}(\cdot)\right) \nabla Z_{T}^{t, \xi}+\int_{s}^{T} \nabla F\left(r, Z_{r}^{t, \xi}(\cdot)\right) \nabla Z_{r}^{t, \xi} d r \\
-\sqrt{2 \nu} \int_{s}^{T} \nabla X_{r}^{t, \xi} d W_{r} .
\end{array}\right.
$$

Now we assume that the solution $\left(Z_{s}^{t, \xi}, Y_{s}^{t, \xi}, X_{s}^{t, \xi}\right)$ is known, and therefore the FBSDEs (20) are regarded as a system of SDEs with random coefficients. Clearly, if we prove the existence of solution $\left(\nabla Z_{s}^{t, \xi}, \nabla Y_{s}^{t, \xi}, \nabla X_{s}^{t, \xi}\right)$ to (20) it would imply that the solution $\left(Z_{s}^{t, \xi}, Y_{s}^{t, \xi}, X_{s}^{t, \xi}\right)$ is differentiable in $\theta$, and solves (5), (6) in $H^{1}\left(\mathbb{T}^{n}, \mathbb{R}^{n}\right)$. The proof of this fact uses standard approaches described for example in [D] or [B]. The same argument as before applied to the triple $\left(\nabla Z_{s}^{t, \xi}, \nabla Y_{s}^{t, \xi}, \nabla X_{s}^{t, \xi}\right)$ as well as the boundedness of $\nabla h$ and $\nabla F$ on $\mathbb{T}^{n}$ and resp. on $[0, T] \times \mathbb{T}^{n}$ imply the existence and uniqueness of a solution to (20), and therefore the existence and uniqueness of a solution to (5), (6) with respect to the $H^{1}\left(\mathbb{T}^{n}, \mathbb{R}^{n}\right)$-norm. Indeed, consider the map

$$
\Gamma^{(1)}: L_{2}\left(\Omega, L_{2}\left(\mathbb{T}^{n}, \mathbb{R}^{n^{2}}\right)\right) \rightarrow L_{2}\left(\Omega, L_{2}\left(\mathbb{T}^{n}, \mathbb{R}^{n^{2}}\right)\right), \nabla Y_{s}^{t, \xi} \rightarrow \overline{\nabla Y_{s}^{t, \xi}}
$$

which is defined by the FBSDEs:

$$
\begin{aligned}
& \overline{\nabla Z_{s}^{t, \xi}}=\nabla \xi+\int_{t}^{s} \nabla Y_{r}^{t, \xi} d r \\
& \overline{\nabla Y_{s}^{t, \xi}}=\nabla h\left(Z_{T}^{t, \xi}(\cdot)\right) \overline{\nabla Z_{T}^{t, \xi}}+\int_{s}^{T} \nabla F\left(r, Z_{r}^{t, \xi}(\cdot)\right) \overline{\nabla Z_{r}^{t, \xi}} d r \\
& -\sqrt{2 \nu} \int_{s}^{T} \overline{\nabla X_{r}^{t, \xi}} d W_{r} .
\end{aligned}
$$

The process $\overline{\nabla Z_{s}^{t, \xi}}$ is obtained from (21), and the processes $\overline{\nabla Y_{s}^{t, \xi}}$ and $\overline{\nabla X_{s}^{t, \xi}}$ are obtained from the BSDE $(22)$ as its unique $\mathcal{F}_{s}$-adapted solution. Let $\nabla V_{s}^{t, \xi}, \overline{\nabla U_{s}^{t, \xi}}, \overline{\nabla V_{s}^{t, \xi}}$, and $\overline{\nabla W_{s}^{t, \xi}}$ be associated with the map $\Gamma^{(1)}$ and correspond to the processes $V_{s}^{t, \xi}, \bar{U}_{s}^{t, \xi}, \bar{V}_{s}^{t, \xi}$, and $\bar{W}_{s}^{t, \xi}$ in the fixed point argument for the map $\Gamma$. The SDE (21) implies the estimate:

$$
\left\|\overline{\nabla Z_{s}^{t, \xi}}-\overline{\nabla U_{s}^{t, \xi}}\right\|_{L_{2}\left(\mathbb{T}^{n}, \mathbb{R}^{\left.n^{2}\right)}\right.}^{2} \leqslant(s-t) \int_{t}^{s}\left\|\nabla Y_{r}^{t, \xi}-\nabla V_{r}^{t, \xi}\right\|_{L_{2}\left(\mathbb{T}^{n}, \mathbb{R}^{n^{2}}\right)}^{2} d r
$$


Application of Itô's formula to $\left\|\overline{\nabla Y_{r}^{t, \xi}}-\overline{\nabla V_{r}^{t, \xi}}\right\|_{L_{2}\left(\mathbb{T}^{n}, \mathbb{R}^{\left.n^{2}\right)}\right.}^{2}$ gives

$$
\begin{aligned}
& \mathbb{E}\left\|\overline{\nabla Y_{s}^{t, \xi}}-\overline{\nabla V_{s}^{t, \xi}}\right\|_{L_{2}\left(\mathbb{T}^{n}, \mathbb{R}^{n^{2}}\right)}^{2}+2 \nu \int_{s}^{T} \mathbb{E}\left\|\overline{\nabla X_{r}^{t, \xi}}-\overline{\nabla W_{r}^{t, \xi}}\right\|_{L_{2}\left(\mathbb{T}^{n}, \mathbb{R}^{n^{3}}\right)}^{2} \\
& =\mathbb{E}\left\|\nabla h\left(Z_{T}^{t, \xi}(\cdot)\right)\left(\overline{\nabla Z_{T}^{t, \xi}}-\overline{\nabla U_{T}^{t, \xi}}\right)\right\|_{L_{2}\left(\mathbb{T}^{n}, \mathbb{R}^{n^{2}}\right)}^{2} \\
& +2 \int_{s}^{T} \mathbb{E}\left(\nabla F\left(r, Z_{r}^{t, \xi}(\cdot)\right)\left(\overline{\nabla Z_{r}^{t, \xi}}-\overline{\nabla U_{r}^{t, \xi}}\right), \overline{\nabla Y_{r}^{t, \xi}}-\overline{\nabla V_{r}^{t, \xi}}\right)_{L_{2}\left(\mathbb{T}^{n}, \mathbb{R}^{n^{2}}\right)} .
\end{aligned}
$$

The same argument as for the map $\Gamma$ implies that

$$
\sup _{s \in[t, T]} \mathbb{E}\left\|\overline{\nabla Y_{s}^{t, \xi}}-\overline{\nabla V_{s}^{t, \xi}}\right\|_{L_{2}\left(\mathbb{T}^{n}, \mathbb{R}^{n^{2}}\right)}^{2} \leqslant \gamma(T) \sup _{s \in[t, T]} \mathbb{E}\left\|\nabla Y_{s}^{t, \xi}-\nabla V_{s}^{t, \xi}\right\|_{L_{2}\left(\mathbb{T}^{n}, \mathbb{R}^{n^{2}}\right)}^{2}
$$

where $\gamma(T)$ and $T$ can be choosen in exactly the same as in (19). Now let us assume that we proved the existence of solution to (5), (6) in $H^{l-1}\left(\mathbb{T}^{n}, \mathbb{R}^{n}\right)$. Namely, we formally differentiate (5), (6) $l-1$ times with respect to $\theta$, and assume that we have proved the existence of a solution $\left(\nabla^{l-1} Z_{s}^{t, \xi}, \nabla^{l-1} Y_{s}^{t, \xi}, \nabla^{l-1} X_{s}^{t, \xi}\right)$ in the space $L_{2}\left(\mathbb{T}^{n}, \mathbb{R}^{n^{l-1}}\right)$. Let us differentiate the FBSDE (5), (6) once again. We obtain the FBSDEs (11), (12) which we consider as the FBSDEs in $L_{2}\left(\mathbb{T}^{n}, \mathbb{R}^{n^{l}}\right)$ with random coefficients with respect to three unknown processes $\left(\nabla^{l} Z_{s}^{t, \xi}, \nabla^{l} Y_{s}^{t, \xi}, \nabla^{l} X_{s}^{t, \xi}\right)$. Consider the map

$$
\Gamma^{(l)}: L_{2}\left(\Omega, L_{2}\left(\mathbb{T}^{n}, \mathbb{R}^{n^{l}}\right)\right) \rightarrow L_{2}\left(\Omega, L_{2}\left(\mathbb{T}^{n}, \mathbb{R}^{n^{l}}\right)\right), \nabla^{l} Y_{s}^{t, \xi} \rightarrow \overline{\nabla^{l} Y_{s}^{t, \xi}}
$$

which is defined as follows: first we determine $\overline{\nabla^{l} Z_{s}^{t, \xi}}$ from the SDE

$$
\overline{\nabla^{l} Z_{s}^{t, \xi}}=\nabla^{l} \xi+\int_{t}^{s} \nabla^{l} Y_{r}^{t, \xi} d r
$$

Then we substitute $\overline{\nabla^{l} Z_{s}^{t, \xi}}$ in the SDE

$$
\begin{aligned}
& \overline{\nabla^{l} Y_{s}^{t, \xi}}=\nabla h\left(Z_{T}^{t, \xi}(\cdot)\right) \overline{\nabla^{l} Z_{T}^{t, \xi}}+\int_{s}^{T} \nabla F\left(r, Z_{r}^{t, \xi}(\cdot)\right) \overline{\nabla^{l} Z_{r}^{t, \xi}} d r \\
& +\sqrt{2 \nu} \int_{s}^{T} \overline{\nabla^{l} X_{r}^{t, \xi}} d W_{r}+\sum_{j=2}^{l} \nabla^{j} h\left(Z_{T}^{t, \xi}(\cdot)\right)\left[\sum_{i_{1}+\cdots+i_{j}=l-j+1} \nabla^{i_{1}} Z_{T}^{t, \xi} \ldots \nabla^{i_{j}} Z_{T}^{t, \xi}\right] \\
& +\int_{s}^{T} \sum_{j=2}^{l} \nabla^{j} F\left(r, Z_{r}^{t, \xi}(\cdot)\right)\left[\sum_{i_{1}+\cdots+i_{j}=l-j+1} \nabla^{i_{1}} Z_{r}^{t, \xi} \ldots \nabla^{i_{j}} Z_{r}^{t, \xi}\right] d r
\end{aligned}
$$


and find a couple $\left(\overline{\nabla^{l} Y_{s}^{t, \xi}}, \overline{\nabla^{l} X_{s}^{t, \xi}}\right)$ as the unique $\mathcal{F}_{s^{-}}$adapted solution to (23). Namely, we have the following expression for $\overline{\nabla^{l} Y_{s}^{t, \xi}}$ :

$$
\begin{aligned}
& \overline{\nabla^{l} Y_{s}^{t, \xi}}=\mathbb{E}\left[\nabla h\left(Z_{T}^{t, \xi}(\cdot)\right) \overline{\nabla^{l} Z_{T}^{t, \xi}}+\int_{s}^{T} \nabla F\left(r, Z_{r}^{t, \xi}(\cdot)\right) \overline{\nabla^{l} Z_{r}^{t, \xi}} d r\right. \\
& +\sum_{j=2}^{l} \nabla^{j} h\left(Z_{T}^{t, \xi}(\cdot)\right)\left[\sum_{i_{1}+\cdots+i_{j}=l-j+1} \nabla^{i_{1}} Z_{T}^{t, \xi} \ldots \nabla^{i_{j}} Z_{T}^{t, \xi}\right] \\
& \left.+\int_{s}^{T} \sum_{j=2}^{l} \nabla^{j} F\left(r, Z_{r}^{t, \xi}(\cdot)\right)\left[\sum_{i_{1}+\cdots+i_{j}=l-j+1} \nabla^{i_{1}} Z_{r}^{t, \xi} \ldots \nabla^{i_{j}} Z_{r}^{t, \xi}\right] d r \mid \mathcal{F}_{s}\right] .
\end{aligned}
$$

By Lemma 1, the last two terms of (24) belong to $L_{2}\left(\mathbb{T}^{n}, \mathbb{R}^{n^{l}}\right)$, and therefore $\overline{\nabla^{l} Y_{s}^{t, \xi}}$ takes values in $L_{2}\left(\mathbb{T}^{n}, \mathbb{R}^{n^{l}}\right)$. As before, we find $\overline{\nabla^{l} X_{r}^{t, \xi}}$ by the martingale representation theorem. Since the coefficients of $\nabla^{l} Z_{T}^{t, \xi}$ and of $\nabla^{l} Y_{r}^{t, \xi}$ under the integral sign are the same as in the case $l=1$, the fixed point argument will be also the same as for this case. In particular, $T_{0}$ will be the same as for the FBSDEs (20) and (5), (6). By induction, we conclude that (11), (12) has $\mathcal{F}_{s}$-adapted solutions $\left(\nabla^{l} Z_{s}^{t, \xi}, \nabla^{l} Y_{s}^{t, \xi}, \nabla^{l} X_{s}^{t, \xi}\right)$ for every $l=1, \ldots, \alpha$. This implies that there exists an $\mathcal{F}_{s}$-adapted $H^{\alpha}\left(\mathbb{T}^{n}, \mathbb{R}^{n}\right) \times H^{\alpha}\left(\mathbb{T}^{n}, \mathbb{R}^{n}\right) \times$ $H^{\alpha}\left(\mathbb{T}^{n}, \mathbb{R}^{n \times n}\right)$-solution to (5), (6).

Uniqueness of solution can be shown as in the proof of Lemma 15 of [C-S].

We have now shown the existence of solution for the forward-backward system of stochastic equations (2). From here we proceed to obtain the deterministic function $y$ which actually determines the drift of the process $Z_{s}^{t, e}$. This procedure is the same that we have followed in [C-S] to derive the solution of Navier-Stokes equations from the solution of the corresponding stochastic system. The difference is that, since now we are dealing with Burgers equation the incompressibility condition ( $\operatorname{div} y=0)$ is not present and, accordingly, the process $Z_{s}^{t, e}$ here belongs to the group $G^{\alpha}$ of $H^{\alpha}$ diffeomorphisms $\mathbb{T}^{n} \rightarrow \mathbb{T}^{n}$ whereas in $[\mathrm{C}-\mathrm{S}]$ the corresponding relevant space is the subgroup of the volume-preserving diffeomorphisms. Still, for sake of completeness, we present here the rest of the proof. Everywhere below we assume that $T<T_{0}$, where $T_{0}$ is defined in Lemma 2 . 
Lemma 3. If $\xi$ is an $\mathcal{F}_{t}$-measurable $H^{\alpha}\left(\mathbb{T}^{n}, \mathbb{R}^{n}\right)$-valued random variable, then the solution $\left(Z_{s}^{t, \xi}, Y_{s}^{t, \xi}, X_{s}^{t, \xi}\right)$ to $(5)$, (6) takes the form:

$$
\left(Z_{s}^{t, \xi}, Y_{s}^{t, \xi}, X_{s}^{t, \xi}\right)=\left(Z_{s}^{t, e} \circ \xi, Y_{s}^{t, e} \circ \xi, X_{s}^{t, e} \circ \xi\right) .
$$

Proof. It suffices to prove the statement of the lemma in the space $L_{2}\left(\mathbb{T}^{n}, \mathbb{R}^{n}\right)$. Indeed, by uniqueness of solution, $\left(Z_{s}^{t, \xi}, Y_{s}^{t, \xi}, X_{s}^{t, \xi}\right)$ is the unique solution to (5), (6) in $L_{2}\left(\mathbb{T}^{n}, \mathbb{R}^{n}\right)$, and therefore if we prove $(25)$ in $L_{2}\left(\mathbb{T}^{n}, \mathbb{R}^{n}\right)$, then the triple $\left(Z_{s}^{t, e} \circ \xi, Y_{s}^{t, e} \circ \xi, X_{s}^{t, e} \circ \xi\right)$ is the unique solution to (5), (6) also in $H^{\alpha}\left(\mathbb{T}^{n}, \mathbb{R}^{n}\right)$.

Let us prove the statement in $L_{2}\left(\mathbb{T}^{n}, \mathbb{R}^{n}\right)$. We apply the operator $R_{\xi}$ of the composition with $\xi$ to both parts of the SDEs (26) and (27):

$$
\begin{aligned}
Z_{s}^{t, e} & =e+\int_{t}^{s} Y_{r}^{t, e} d r+\sqrt{2 \nu}\left(W_{s}-W_{t}\right), \\
Y_{s}^{t, e} & =h \circ Z_{T}^{t, e}+\int_{s}^{T} F(r, \cdot) \circ Z_{r}^{t, e} d r-\sqrt{2 \nu} \int_{s}^{T} X_{r}^{t, e} d W_{r} .
\end{aligned}
$$

Let us observe that we can write $R_{\xi}$ under the integral signs of the Bochner integrals. Indeed, since these integrals converge in $H^{\alpha}\left(\mathbb{T}^{n}, \mathbb{R}^{n}\right)$, they also converge with respect to at least the $\mathrm{C}\left(\mathbb{T}^{n}, \mathbb{R}^{n}\right)$-topology. Due to the periodicity of the functions under the (Bochner) integrals signs, the composition of the integrands with $\xi$ will preserve the convergence with respect to the $\mathrm{C}\left(\mathbb{T}^{n}, \mathbb{R}^{n}\right)$-topology. For the integrals converging in the $\mathrm{C}\left(\mathbb{T}^{n}, \mathbb{R}^{n}\right)$-topology, we can easily see that we are allowed to write $R_{\xi}$ under the integral signs. This implies that if we consider convergence of the Bochner integrals with respect to the $L_{2}\left(\mathbb{T}^{n}, \mathbb{R}^{n}\right)$-topology, we can also write $R_{\xi}$ under the integral signs.

Let us prove now that we are allowed to write $R_{\xi}$ under the integral signs of the stochastic integrals in (26), (27). First we prove this for the case when $\xi=g$ is deterministic. Let $s$ and $S$ be such that $t \leqslant s<S \leqslant T$, and let $\Phi_{r}$ be an $\mathcal{F}_{r}$-adapted stochastically integrable process, and let $I\left(\Phi_{r}\right)$ denote $\int_{s}^{S} \Phi_{r} d W_{r}$. Let $\Phi_{r}^{(m)}$ be a sequence of simple stochastic processes such that $I\left(\Phi_{r}^{(m)}\right)$ converge to $I\left(\Phi_{r}\right)$ with respect to the $L_{2}(\Omega) \times H^{\alpha}\left(\mathbb{T}^{n}, \mathbb{R}^{n}\right)$-norm. Note that if $F \in H^{\alpha}\left(\mathbb{T}^{n}, \mathbb{R}^{n}\right)$ extended to $\mathbb{R}^{n}$ periodically, then there exist constants $\gamma_{1}$ and $\gamma_{2}$ not depending on $F$ and such that

$$
\|F \circ g\|_{L_{2}\left(\mathbb{T}^{n}, \mathbb{R}^{n}\right)} \leqslant \gamma_{1}\|F \circ g\|_{\mathrm{C}\left(\mathbb{T}^{n}, \mathbb{R}^{n}\right)} \leqslant \gamma_{1}\|F\|_{\mathrm{C}\left(\mathbb{T}^{n}, \mathbb{R}^{n}\right)} \leqslant \gamma_{2}\|F\|_{H^{\alpha}\left(\mathbb{T}^{n}, \mathbb{R}^{n}\right)} .
$$


Therefore, since $\mathbb{E} \int_{s}^{S}\left\|\Phi_{r}^{(m)}-\Phi_{r}\right\|_{H^{\alpha}\left(\mathbb{T}^{n}, \mathbb{R}^{n}\right)}^{2} d r \rightarrow 0$, then $\mathbb{E} \int_{s}^{S} \| \Phi_{r}^{(m)} \circ g-\Phi_{r} \circ$ $g \|_{L_{2}\left(\mathbb{T}^{n}, \mathbb{R}^{n}\right)}^{2} d r \rightarrow 0$. By Itô's isometry, $\mathbb{E}\left\|I\left(\Phi_{r}^{(m)} \circ g\right)-I\left(\Phi_{r} \circ g\right)\right\|_{L_{2}\left(\mathbb{T}^{n}, \mathbb{R}^{n}\right)} \rightarrow 0$. Again using (28), we conclude that $\mathbb{E}\left\|I\left(\Phi_{r}^{(m)}\right) \circ g-I\left(\Phi_{r}\right) \circ g\right\|_{L_{2}\left(\mathbb{T}^{n}, \mathbb{R}^{n}\right)}^{2} \rightarrow$ 0 because $\mathbb{E}\left\|I\left(\Phi_{r}^{(m)}\right)-I\left(\Phi_{r}\right)\right\|_{H^{\alpha}\left(\mathbb{T}^{n}, \mathbb{R}^{n}\right)}^{2} \rightarrow 0$. Clearly, for simple stochastic processes it holds that $I\left(\Phi_{r}^{(m)}\right) \circ g=I\left(\Phi_{r}^{(m)} \circ g\right)$, and therefore $I\left(\Phi_{r}\right) \circ g=$ $I\left(\Phi_{r} \circ g\right)$.

Now let us take an $\mathcal{F}_{t}$-measurable stepwise function $\xi=\sum_{i=1}^{\infty} g_{i} \mathbb{I}_{A_{i}}$, where $g_{i} \in H^{\alpha}\left(\mathbb{T}^{n}, \mathbb{R}^{n}\right)$ and the sets $A_{i}$ are $\mathcal{F}_{t}$-measurable. We obtain:

$$
\begin{aligned}
& \int_{s}^{S} \Phi_{r} d W_{r} \circ \sum_{i=1}^{\infty} g_{i} \mathbb{I}_{A_{i}}=\sum_{i=1}^{\infty} \mathbb{I}_{A_{i}} \int_{s}^{S} \Phi_{r} \circ g_{i} d W_{r}=\sum_{i=1}^{\infty} \int_{s}^{S} \mathbb{I}_{A_{i}} \Phi_{r} \circ g_{i} d W_{r} \\
& =\int_{s}^{S} \Phi_{r} \circ \sum_{i=1}^{\infty} g_{i} \mathbb{I}_{A_{i}} d W_{r}
\end{aligned}
$$

Next, we find a sequence of $\mathcal{F}_{t}$-measurable stepwise functions converging to $\xi$ in the space of continuous functions $\mathrm{C}\left(\mathbb{T}^{n}, \mathbb{R}^{n}\right)$. This is possible due to the separability of $\mathrm{C}\left(\mathbb{T}^{n}, \mathbb{R}^{n}\right)$. Indeed, let us consider a countable number of disjoint Borel sets $O_{i}^{n}$ covering $\mathrm{C}\left(\mathbb{T}^{n}, \mathbb{R}^{n}\right)$, and such that their diameter in the norm of $\mathrm{C}\left(\mathbb{T}^{n}, \mathbb{R}^{n}\right)$ is smaller than $\frac{1}{n}$. Let $A_{i}^{n}=\xi^{-1}\left(O_{i}^{n}\right)$ and $g_{i}^{n} \in O_{i}^{n}$. Define $\xi_{n}=\sum_{i=1}^{\infty} g_{i}^{n} \mathbb{I}_{A_{i}^{n}}$. Then for all $\omega \in \Omega$, we have $\left\|\xi-\xi_{n}\right\|_{\mathrm{C}\left(\mathbb{T}^{n}, \mathbb{R}^{2}\right)}<\frac{1}{n}$. As before, $I(\Phi)$ and $I(\Phi \circ \xi)$ denote $\int_{s}^{S} \Phi_{r} d W_{r}$ and $\int_{s}^{S} \Phi_{r} \circ \xi d W_{r}$ resp. We have to prove that a.s. $I(\Phi) \circ \xi=I(\Phi \circ \xi)$. For this it suffices to prove that

$$
\begin{aligned}
& \lim _{n \rightarrow \infty} \mathbb{E}\left\|I(\Phi) \circ \xi_{n}-I(\Phi) \circ \xi\right\|_{L_{2}\left(\mathbb{T}^{n}, \mathbb{R}^{2}\right)}^{2}=0, \\
& \lim _{n \rightarrow \infty} \mathbb{E}\left\|I\left(\Phi \circ \xi_{n}\right)-I(\Phi \circ \xi)\right\|_{L_{2}\left(\mathbb{T}^{n}, \mathbb{R}^{2}\right)}=0 .
\end{aligned}
$$

By (28), $\left\|I(\Phi) \circ \xi_{n}\right\|_{L_{2}\left(\mathbb{T}^{n}, \mathbb{R}^{n}\right)} \leqslant \gamma_{2}\|I(\Phi)\|_{H^{\alpha}\left(\mathbb{T}^{n}, \mathbb{R}^{n}\right)}$, and $\|I(\Phi) \circ \xi\|_{L_{2}\left(\mathbb{T}^{n}, \mathbb{R}^{n}\right)} \leqslant$ $\gamma_{2}\|I(\Phi)\|_{H^{\alpha}\left(\mathbb{T}^{n}, \mathbb{R}^{n}\right)}$. By Lebesgue's theorem, in (29) we can pass to the limit under the expectation sign. Relation (29) holds then by the continuity of $I(\Phi)$ in $\theta \in \mathbb{T}^{n}$. To prove (30) we observe that by Itô's isometry, the limit in (30) equals to $\lim _{n \rightarrow \infty} \mathbb{E} \int_{s}^{S}\left\|\Phi_{r} \circ \xi_{n}-\Phi_{r} \circ \xi\right\|_{L_{2}\left(\mathbb{T}^{n}, \mathbb{R}^{2}\right)}^{2} d r$. The same argument that we used to prove (29) implies that we can pass to the limit under the expectation and the integral signs. Relation (30) follows from the continuity of $\Phi_{r}$ in $\theta \in \mathbb{T}^{n}$. Hence, $\left(Z_{s}^{t, e} \circ \xi, Y_{s}^{t, e} \circ \xi, X_{s}^{t, e} \circ \xi\right)$ is a solution to (5), (6) in $L_{2}\left(\mathbb{T}^{n}, \mathbb{R}^{n}\right)$. 
Lemma 4. The processes $Z_{s}^{t, e}$ and $Y_{s}^{t, e}$ have continuous path modifications. Namely, for these modifications it holds that the trajectories $[t, T] \rightarrow$ $L_{2}\left(\mathbb{T}^{n}, \mathbb{R}^{n}\right), s \mapsto Z_{s}^{t, e}$ and $[t, T] \rightarrow L_{2}\left(\mathbb{T}^{n}, \mathbb{R}^{n}\right), s \mapsto Y_{s}^{t, e}$ are continuous with probability 1.

Proof. Let $s^{\prime}>s$. Application of Itô's formula together with the BSDE (27) imply:

$$
\left\|Y_{s}^{t, e}-Y_{s^{\prime}}^{t, e}\right\|_{L_{2}\left(\mathbb{T}^{n}, \mathbb{R}^{n}\right)}^{2} \leqslant \int_{s}^{s^{\prime}}\left\|F\left(r, Z_{r}^{t, e}\right)\right\|_{L_{2}\left(\mathbb{T}^{n}, \mathbb{R}^{n}\right)}^{2} d r+\int_{s}^{s^{\prime}}\left\|Y_{r}^{t, e}-Y_{s^{\prime}}^{t, e}\right\|_{L_{2}\left(\mathbb{T}^{n}, \mathbb{R}^{n}\right)}^{2} d r .
$$

Gronwall's lemma implies that there exist constants $\tilde{\gamma}>0$ and $\gamma>0$ such that

$$
\left\|Y_{s}^{t, e}-Y_{s^{\prime}}^{t, e}\right\|_{L_{2}\left(\mathbb{T}^{n}, \mathbb{R}^{n}\right)}^{2} \leqslant \tilde{\gamma} \int_{s}^{s^{\prime}}\left\|F\left(r, Z_{r}^{t, e}\right)\right\|_{L_{2}\left(\mathbb{T}^{n}, \mathbb{R}^{n}\right)}^{2} d r \leqslant \gamma\left(s^{\prime}-s\right) .
$$

This implies that if $p>1$ then

$$
\left\|Y_{s}^{t, e}-Y_{s^{\prime}}^{t, e}\right\|_{L_{2}\left(\mathbb{T}^{n}, \mathbb{R}^{n}\right)}^{2 p} \leqslant \gamma\left|s-s^{\prime}\right|^{p} .
$$

By Kolmogorov's continuity criteria, $Y_{s}^{t, e}$ has a continuous path modification with respect to the $L_{2}\left(\mathbb{T}^{n}, \mathbb{R}^{n}\right)$-topology. The SDE (26) implies that $Z_{s}^{t, e}$ has a continuous path modification in the $L_{2}\left(\mathbb{T}^{n}, \mathbb{R}^{n}\right)$-topology as well.

Lemmas 5 and 6 below characterize the deterministic nature of the process $Y_{s}^{t, e}$ and describe its continuity properties.

LEMma 5. The map

$$
[0, T] \times \mathbb{T}^{n} \rightarrow \mathbb{R}^{n},(t, \theta) \rightarrow Y_{t}^{t, e}(\theta)
$$

is deterministic and the function $[0, T] \rightarrow H^{\alpha}\left(\mathbb{T}^{n}, \mathbb{R}^{n}\right), t \mapsto Y_{t}^{t, e}$ is continuous.

Proof. The first statement is a consequence of Blumenthal's zero-one law and the fact that the random variable $Y_{t}^{t, e}$ is $\mathcal{F}_{0}$-measurable (as in Lemma 13 of [C-S] or Corollary 1.5. of [D]).

The proof of the continuity of the map $[0, T] \rightarrow L_{2}\left(\mathbb{T}^{n}, \mathbb{R}^{n}\right), t \mapsto Y_{t}^{t, e}$ follows as in Lemma 14 of [C-S]. Consider the FBSDEs below on the interval 
$[0, T]$ with respect to $\left(\nabla^{l} Z_{s}^{t, \xi}, \nabla^{l} Y_{s}^{t, \xi}, \nabla^{l} X_{s}^{t, \xi}\right)$ :

$$
\begin{aligned}
& \nabla^{l} Z_{s}^{t, \xi}=\nabla^{l} \xi+\int_{0}^{s} \mathbb{I}_{[t, T]} \nabla^{l} Y_{r}^{t, \xi} d r \\
& \nabla^{l} Y_{s}^{t, \xi}=\nabla h\left(Z_{T}^{t, \xi}(\cdot)\right) \nabla^{l} Z_{T}^{t, \xi}+\int_{s}^{T} \mathbb{I}_{[t, T]} \nabla F\left(r, Z_{r}^{t, \xi}(\cdot)\right) \nabla^{l} Z_{r}^{t, \xi} d r \\
& +\sum_{j=2}^{l} \nabla^{j} h\left(Z_{T}^{t, \xi}(\cdot)\right)\left[\sum_{i_{1}+\cdots+i_{j}=l-j+1} \nabla^{i_{1}} Z_{T}^{t, \xi} \ldots \nabla^{i_{j}} Z_{T}^{t, \xi}\right] \\
& +\int_{s}^{T} \mathbb{I}_{[t, T]} \sum_{j=2}^{l} \nabla^{j} F\left(r, Z_{r}^{t, \xi}(\cdot)\right)\left[\sum_{i_{1}+\cdots+i_{j}=l-j+1} \nabla^{i_{1}} Z_{r}^{t, \xi} \ldots \nabla^{i_{j}} Z_{r}^{t, \xi}\right] d r \\
& -\sqrt{2 \nu} \int_{s}^{T} \nabla^{l} X_{r}^{t, \xi} d W_{r}
\end{aligned}
$$

and note that its solution $\left(\nabla^{l} Z_{s}^{t, \xi}, \nabla^{l} Y_{s}^{t, \xi}, \nabla^{l} X_{s}^{t, \xi}\right)$ can be obtained from the solution to (11), (12) by extending it to $[0, t]$ as follows: $\nabla^{l} Z_{s}^{t, \xi}=\nabla^{l} \xi$, $\nabla^{l} Y_{s}^{t, \xi}=\nabla^{l} Y_{t}^{t, \xi}, \nabla^{l} X_{s}^{t, \xi}=0, s \in[0, t]$. The extended triple solves the FBSDEs $(31),(32)$ on $[0, T]$. The same argument as in the proof of Lemma 14 of $[\mathrm{C}-\mathrm{S}]$ implies that there exists a constant $\gamma>0$ such that

$$
\left\|\nabla^{l} Y_{t}^{t, \xi}-\nabla^{l} Y_{t^{\prime}}^{t^{\prime}, \xi}\right\|_{L_{2}\left(\mathbb{T}^{n}, \mathbb{R}^{n^{l}}\right)} \leqslant \gamma\left|t-t^{\prime}\right|
$$

Therefore the map $t \mapsto Y_{t}^{t, \xi}$ is continuous with respect to the $H^{\alpha}\left(\mathbb{T}^{n}, \mathbb{R}^{n}\right)$ topology.

Lemma 6. Let the function $y:[0, T] \times \mathbb{T}^{n} \rightarrow \mathbb{R}^{n}$ be defined by the formula

$$
y(t, \theta)=Y_{t}^{t, e}(\theta) .
$$

Then, for every $t \in[0, T]$, there exists a set $\Omega^{\prime} \subset \Omega$ of full $\mathbb{P}$-measure, so that for all $u \in[t, T]$, for all $\omega \in \Omega^{\prime}$ the following relation holds:

$$
Y_{u}^{t, e}=y(u, \cdot) \circ Z_{u}^{t, e} .
$$

Proof. Note that (25) implies that if $\xi$ is $\mathcal{F}_{t}$-measurable then

$$
Y_{t}^{t, \xi}=y(t, \cdot) \circ \xi
$$


Further, for each fixed $u \in[t, T],\left(Z_{s}^{t, e}, Y_{s}^{t, e}, X_{s}^{t, e}\right)$ is a solution of the following problem on $[u, T]$ :

$$
\left\{\begin{array}{l}
Z_{s}^{t, e}=Z_{u}^{t, e}+\int_{u}^{s} Y_{r}^{t, e} d r+\sqrt{2 \nu}\left(W_{s}-W_{u}\right), \\
Y_{s}^{t, e}=h\left(Z_{T}^{t, e}(\cdot)\right)+\int_{s}^{T} F\left(r, Z_{r}^{t, e}(\cdot)\right) d r-\sqrt{2 \nu} \int_{s}^{T} X_{r}^{t, e} d W_{r} .
\end{array}\right.
$$

By the uniqueness of solution, it holds that $Y_{s}^{t, e}=Y_{s}^{u, Z_{u}^{t, e}}$ a.s. on $[u, T]$. Next, by (35), we obtain that $Y_{u}^{u, Z_{u}^{t, e}}=y(u, \cdot) \circ Z_{u}^{t, e}$. This implies that there exists a set $\Omega_{u}$ (which depends on $u$ ) of full $\mathbb{P}$-measure such that (34) holds everywhere on $\Omega_{u}$. Clearly, one can find a set $\Omega_{\mathbb{Q}}, \mathbb{P}\left(\Omega_{\mathbb{Q}}\right)=1$, such that (34) holds on $\Omega_{\mathbb{Q}}$ for all rational $u \in[t, T]$. But the trajectories of $Z_{s}^{t, e}$ and $Y_{s}^{t, e}$ are a.s. continuous with respect to $L_{2}\left(\mathbb{T}^{n}, \mathbb{R}^{n}\right)$-topology by Lemma 4 . Furthermore $y(t, \cdot)$ is continuous in $t$ with respect to (at least) the $L_{2}\left(\mathbb{T}^{n}, \mathbb{R}^{n}\right)$-topology. Therefore, (34) holds a.s. with respect to the $L_{2}\left(\mathbb{T}^{n}, \mathbb{R}^{n}\right)$-topology. Since both sides of (34) are continuous in $\theta \in \mathbb{T}^{n}$ it also holds a.s. for all $\theta \in \mathbb{T}^{n}$.

Finally the function $y(s, \cdot)$ defined by (33) indeed verifies the Burgers equation. This is the content of the next lemma.

LEMma 7. The function y defined by formula (33) is $C^{1}$-smooth in $t \in[0, T]$, and is a solution of problem (1).

Proof. Let $\delta>0$. We obtain:

$$
y(t+\delta, \cdot)-y(t, \cdot)=Y_{t+\delta}^{t+\delta, e}-Y_{t}^{t, e}=Y_{t+\delta}^{t+\delta, e}-Y_{t+\delta}^{t, e}+Y_{t+\delta}^{t, e}-Y_{t}^{t, e} .
$$

As before, let $G^{\alpha}$ be the group of $H^{\alpha}$-diffeomorphisms $\mathbb{T}^{n} \rightarrow \mathbb{T}^{n}$, and let $\hat{Y}_{s}$ be the right-invariant vector field on $G^{\alpha}$ generated by $y(s, \cdot)$ (see $[\mathrm{C}-\mathrm{S}]$ ). Relation (34) allows us to represent the SDE (26) as an SDE on the manifold $G^{\alpha}$. Indeed, by results of [G1] and [C-S], the SDE

$$
\left\{\begin{array}{l}
d Z_{s}^{t, e}=\exp \left\{\hat{Y}_{s}\left(Z_{s}^{t, e}\right) d s+\sqrt{2 \nu} d W_{s}\right\} \\
Z_{t}^{t, e}=e
\end{array}\right.
$$

where exp is the exponential map of the weak Riemannian metric on $G^{\alpha}$ (see $[\mathrm{C}-\mathrm{S}]$ ), has a unique $G^{\alpha}$-valued solution. As it was proved in [C-S], the latter solution coincides with the unique solution of the $H^{\alpha}\left(\mathbb{T}^{n}, \mathbb{R}^{n}\right)$-valued SDE

$$
\left\{\begin{array}{l}
d Z_{s}^{t, e}=y(s, \cdot) \circ Z_{s}^{t, e} d s+\sqrt{2 \nu} d W_{s}, \\
Z_{t}^{t, e}=e
\end{array}\right.
$$


Therefore, the $Z_{s}^{t, e}$-part of the solution to (26), (27) is the unique solution to (36). By Lemma 6, a.s. $Y_{t+\delta}^{t, e}=\hat{Y}_{t+\delta}\left(Z_{t+\delta}^{t, e}\right)$. Thus we obtain that a.s.

$$
y(t+\delta, \cdot)-y(t, \cdot)=\left(\hat{Y}_{t+\delta}(e)-\hat{Y}_{t+\delta}\left(Z_{t+\delta}^{t, e}\right)\right)+\left(Y_{t+\delta}^{t, e}-Y_{t}^{t, e}\right) .
$$

We use the BSDE (27) for the second difference and apply Itô's formula to the first difference when considering $\hat{Y}_{t+\delta}$ as a $C^{2}$-smooth function $G^{\alpha} \rightarrow$ $L_{2}\left(\mathbb{T}^{n}, \mathbb{R}^{2}\right)$. We obtain:

$$
\begin{aligned}
& \hat{Y}_{t+\delta}\left(Z_{t+\delta}^{t, e}\right)-\hat{Y}_{t+\delta}(e)=\int_{t}^{t+\delta} \hat{Y}_{r}^{t, e}\left(Z_{r}^{t, e}\right)\left[\hat{Y}_{t+\delta}\left(Z_{r}^{t, e}\right)\right] d r \\
& \quad+\sqrt{2 \nu} \int_{t}^{t+\delta} \sum_{i=1}^{n}\left[\bar{\nabla}_{e_{i}} \hat{Y}_{t+\delta}\left(Z_{r}^{t, e}\right)\right] d W_{r}+2 \nu \int_{t}^{t+\delta} \sum_{i=1}^{n}\left[\bar{\nabla}_{e_{i}}^{2} \hat{Y}_{t+\delta}\left(Z_{r}^{t, e}\right)\right] d r
\end{aligned}
$$

where $\bar{\nabla}$ is the covariant derivative on $G^{\alpha}, e_{i}$ are regarded as constant vector fields on $G^{\alpha}$, and the expression $\hat{Y}_{r}^{t, e}\left(Z_{r}^{t, e}\right)\left[\hat{Y}_{t+\delta}\left(Z_{r}^{t, e}\right)\right]$ has the same meaning as in $[\mathrm{C}-\mathrm{S}]$. We obtain:

$$
\begin{aligned}
& \hat{Y}_{t+\delta}\left(Z_{t+\delta}^{t, e}\right)-\hat{Y}_{t+\delta}(e)=\int_{t}^{t+\delta} d r \nabla_{y(r, \cdot)} y(t+\delta, \cdot) \circ Z_{r}^{t, e} \\
& \quad+\int_{t}^{t+\delta} d r \nu \Delta y(t+\delta, \cdot) \circ Z_{r}^{t, e}+\sqrt{2 \nu} \int_{t}^{t+\delta} \sum_{i=1}^{n}\left[\bar{\nabla}_{e_{i}} \hat{Y}_{t+\delta}\left(Z_{r}^{t, e}\right)\right] d W_{r} .
\end{aligned}
$$

Further we have:

$$
Y_{t}^{t, e}-Y_{t+\delta}^{t, e}=\int_{t}^{t+\delta} d r F(r, \cdot) \circ Z_{r}^{t, e}-\sqrt{2 \nu} \int_{t}^{t+\delta} X_{r}^{t, e} d W_{r} .
$$

Taking expectations implies:

$$
\begin{aligned}
\frac{1}{\delta}(y(t+\delta, \cdot)-y(t, \cdot))=-\frac{1}{\delta} \mathbb{E}\left[\int_{t}^{t+\delta} d r[(y(r, \cdot), \nabla) y(t+\delta, \cdot)\right. \\
\left.+\nu \Delta y(t+\delta, \cdot)+F(r, \cdot)] \circ Z_{r}^{t, e}\right] .
\end{aligned}
$$

Note that $Z_{r}^{t, e}, F(r, \cdot)$, and $(y(r, \cdot), \nabla) y(t+\delta, \cdot) \circ Z_{r}^{t, e}$ are continuous in $r$ a.s. with respect to the $L_{2}\left(\mathbb{T}^{n}, \mathbb{R}^{2}\right)$-topology. By Lemma $5, \nabla y(t, \cdot)$ and $\Delta y(t, \cdot)$ 
are continuous in $t$ with respect to at least the $L_{2}\left(\mathbb{T}^{n}, \mathbb{R}^{2}\right)$-topology. Formula (37) and the fact that $Z_{t}^{t, e}=e$ imply that in the $L_{2}\left(\mathbb{T}^{n}, \mathbb{R}^{2}\right)$-topology

$$
\partial_{t} y(t, \cdot)=-\left[\nabla_{y(t, \cdot)} y(t, \cdot)+\nu \Delta y(t, \cdot)+F(t, \cdot)\right] \text {. }
$$

Since the right-hand side of (38) is an $H^{\alpha-2}$-map, so is the left-hand side. This implies that $\partial_{t} y(t, \cdot)$ is continuous in $\theta \in \mathbb{T}^{n}$. Therefore, (38) holds for any $\theta \in \mathbb{T}^{n}$. Relation (38) is obtained so far for the right derivative of $y(t, \theta)$ with respect to $t$. Note that the right-hand side of (38) is continuous in $t$ which implies that the right derivative $\partial_{t} y(t, \theta)$ is continuous in $t$ on $[0, T)$. Hence, it is uniformly continuous on every compact subinterval of $[0, T)$. This implies the existence of the left derivative of $y(t, \theta)$ in $t$, and therefore, the existence of the continuous derivative $\partial_{t} y(t, \theta)$ everywhere on $[0, T]$.

REMARK 1. Note that at the same time we have proved that the process $Z_{s}^{t, e}$ takes values in the group $G^{\alpha}$ of $H^{\alpha}$-diffeomorphisms $\mathbb{T}^{n} \rightarrow \mathbb{T}^{n}$.

\section{Acknowledgements}

The first author acknowledges the support of the Portuguese Foundation for Science and Technology through the project PTDC/MAT/69635/2006. The second author acknowledges the support of the Portuguese Foundation for Science and Technology through the Centro de Matemática da Universidade do Porto.

\section{References}

[A] V.I. Arnold, Sur la géométrie différentielle des groupes de Lie de dimension infinie et ses applications a l'hydrodynamique des fluides parfaits, Ann. Inst. Fourier 16 (1966), 316-361.

[B] Ya. I. Belopolskaya, Yu. L. Dalecky, Stochastic equations and differential geometry, Series: Mathematics and its Applications, Kluwer Academic Publishers, Netherlands, (1989), 260 p.

[C-S-T-V] P. Cheridito, H. Mete Soner, N. Touzi and N. Victoir, Second order backward stochastic differential equations and fully nonlinear parabolic PDEs, Comm. Pure Appl. Math. 60 (2007), 1081-1110. 
[C-S] A.B. Cruzeiro, E. Shamarova, Navier-Stokes equations and forwardbackward SDEs on the group of diffeomorphisms of a torus, Stochastic Processes and their Applications, 119, (2009), 4034-4060

[D] F. Delarue, On the existence and uniqueness of solutions to the FBSDEs in a non-generate case, Stoch. Proc. and their Appl. 99, (2002), 209-286.

[G] Yu. E. Gliklikh, Solutions of Burgers, Reynolds, and Navier-Stokes equations via stochastic perturbations of inviscid flows, Journal of Nonlinear Mathematical Physics, Vol. 17, No. Supplementary Issue 1 (2010) 15-29.

[G1] Yu. E. Gliklikh, Global Analysis in Mathematical Physics: Geometric and Stochastic Methods, Springer (1997), 213 p.

[E-M] D.G. Ebin and J. Marsden, Groups of diffeomorphisms and the motion of an incompressible fluid, Ann. of Math. 92 (1970), 102-163.

[N-Y-Z] T. Nakagomi, K. Yasue, J.-C. Zambrini, Stochastic variational derivations of the Navier-Stokes equation, Lett. Math. Phys., 160 (1981), $337-365$.

[Y] K. Yasue, A variational principle for the Navier-Stokes equation,J. Funct. Anal., 51 (2), (1983), 133-141 